\title{
Miranda
}

Revue pluridisciplinaire du monde anglophone /

Multidisciplinary peer-reviewed journal on the English-

speaking world

$20 \mid 2020$

Staging American Nights

\section{Orlando, du collectif GWEN}

Critique

Susan Blattès, Claire Hélie et Anne-Laure Rigeade

\section{OpenEdition}

Journals

Édition électronique

URL : http://journals.openedition.org/miranda/27406

DOI : 10.4000/miranda. 27406

ISSN : 2108-6559

Éditeur

Université Toulouse - Jean Jaurès

Référence électronique

Susan Blattès, Claire Hélie et Anne-Laure Rigeade, «Orlando, du collectif GWEN », Miranda [En ligne], 20 | 2020, mis en ligne le 27 avril 2020, consulté le 16 février 2021. URL : http://

journals.openedition.org/miranda/27406 ; DOI : https://doi.org/10.4000/miranda.27406

Ce document a été généré automatiquement le 16 février 2021.

\section{(c) (i) $\odot$}

Miranda is licensed under a Creative Commons Attribution-NonCommercial-NoDerivatives 4.0 International License. 


\title{
Orlando, du collectif GWEN
}

\author{
Critique
}

Susan Blattès, Claire Hélie et Anne-Laure Rigeade

\section{Informations factuelles sur la pièce}

1 Pièce vue le 16 janvier 2020 au théâtre des Déchargeurs, Paris 1er

2 1h15 sans entracte

3 Adaptation, mise en scène, jeu Lucie Brandsma, Sébastien Dalloni, Thomas Harel

4 Coréalisation La Reine Blanche - les Déchargeurs \& le collectif GWEN

5 Théâtre de La Croisée des Chemins du 11 septembre au 31 octobre 2019

6 Les Déchargeurs du 14 au 30 janvier 2020

\section{Liens}

7 Théâtre Les Déchargeurs : https://www.lesdechargeurs.fr/spectacle/orlando

\section{Critique}

8 Le roman Orlando, de l'écrivaine moderniste Virginia Woolf, a fait l'objet de nombreuses adaptations, que ce soit au cinéma (le film de 1992 de Sally Potter a donné lieu à une performance immersive par des acteurs LGBT en 2017), à l'opéra (Olga Neuwirth et Catherine Filloux ont écrit le livret en anglais pour la production qui a ouvert à Vienne en 2019 avec Kate Lindsey dans le rôle-titre), ou encore au théâtre (de Robert Wilson à Katie Mitchell). Personne n'a peur de Virginia Woolf et le fait que le roman repose sur le jeu et le travestissement favorise sans doute son passage à la scène.

9 Cette fois-ci, ce sont trois jeunes acteurs et actrice au sortir de l'ESCA, l'École Supérieure de Comédiens en alternance d'Asnières, qui s'attaquent à ce roman majeur de 1928. Inspirés par l'adaptation de Robert Wilson et Darry Pinckney, trois des neuf 
membres du collectif GWEN (Lucie Brandsma, Sébastien Dalloni, Thomas Harel) ont écrit, mis en scène et joué une version pour le moins décapante d'Orlando. Ils s'attaquent en effet non seulement au canon littéraire en transposant sur scène leur version du roman, mais aussi au canon théâtral, en modernisant la structure tripartite qu'avait inventée Robert Wilson. Ce faisant, ils font ressortir toute la modernité du texte woolfien et de la direction wilsonienne, et ce notamment grâce à un dispositif scénique efficace, un rythme enlevé et un jeu dynamique.

Figure 1



Lucie Brandsma, Sébastien Dalloni, Thomas Harel dans Orlando. Crédits : Collectif GWEN

\section{Dispositif scénique : jeux avec l'espace, jeux avec le texte}

10 La petite salle de 80 places, remplie aux trois quarts ce soir-là, favorise une proximité avec le public, qui ne peut toutefois à elle seule expliquer l'accueil enthousiaste qui a été fait à la pièce. Les comédiens sont déjà sur le plateau quand le public rentre et, si l'on sent leur fébrilité, le silence s'impose vite de lui-même. Un carré blanc au sol délimite l'espace de jeu et les changements de costume se font à vue, sur les bords, où sont rangés les quelques accessoires qui permettront la traversée du temps et des identités. L'espace scénique rend ainsi visible le dispositif textuel, tant d'Orlando, alternant narration et commentaires sur la narration, que de l'adaptation signée par le collectif GWEN, alternant scènes jouées et scènes commentées par un ou deux acteurs incarnant un biographe-narrateur.

11 Alors que l'adaptation de Robert Wilson (Théâtre de l'Odéon, 21 septembre - 24 octobre 1993) était portée par une seule comédienne en scène (Isabelle Huppert pour la version 
française), ici chacune des trois parties est prise en charge par un des acteurs qui incarne un moment de la vie d'Orlando: le $\mathrm{XVI}^{\mathrm{e}}$ siècle avec sa tonalité légère, quand l'adolescent cherche la voie de l'âge adulte ; les XVII ${ }^{e}$ et XVIII ${ }^{e}$ siècles, quand Orlando se retrouve isolé à la campagne puis pris dans un rythme haletant à Constantinople, où il s'affirme en tant qu'adulte; et enfin les XIX ${ }^{\mathrm{e}}$ et $\mathrm{XX}^{\mathrm{e}}$ siècles, quand Orlando se réveille/ révèle femme. La continuité entre les périodes historiques et les différentes phases de transformation intérieure ou extérieure est apportée par un drap blanc aux multiples fonctions (peignoir, cape ou robe) qui passe d'un acteur à l'autre. Dans chaque partie, ceux qui ne jouent pas Orlando incarnent le biographe-narrateur ou d'autres personnages ou encore font des voix off amplifiées par un micro (il en va ainsi pour Sasha qui n'est jamais représentée sur scène). Loin du «I'm alone » de Wilson, Orlando se donne ainsi comme une expérience théâtrale collective, dans laquelle même les spectateurs ont leur part à jouer, par exemple lorsqu'ils sont invités à taper des mains au rythme des applaudissements enregistrés qui accompagnent l'interview façon talkshow d'Orlando pendant son passage à Constantinople.

L'adaptation restaure ainsi la traversée des siècles occultée dans l'adaptation de Wilson. Mieux, ce sous-texte est mis en exergue car les années, les lieux, les dates historiques importantes comme les règnes des monarques britanniques sont projetés sur l'écran en fond de scène. Le passage du temps est aussi évoqué par le dispositif sonore à travers des répétitions avec variation des Quatre Saisons de Vivaldi. Le temps passe, mais il revient; il se voit, il s'entend.

\section{Modernisation et anachronismes : jeux avec les temps}

De leur propre aveu, les auteurs-adaptateurs n'ont pas cherché à adapter Woolf au goût moderne, mais à faire entendre sa modernité quant à la réflexion menée sur le genre, dans l'acception sociologique du terme.

La troupe a donc écarté tant la dimension réflexive et érudite sur l'écriture historique que la dimension biographique de la liaison de Woolf avec Vita Sackville-West, pour se concentrer sur les enjeux identitaires. De nombreux choix de mise en scène l'attestent, comme par exemple le fait que la reine Elizabeth I soit jouée par un homme portant un masque de femme. De même, dans la troisième partie, l'actrice jouant Orlando fait alterner dans son jeu des poses plus masculines ou plus féminines, comme pour souligner la possibilité de se glisser dans la peau de l'un ou de l'autre sexe à volonté.

L'adaptation scénique met donc l'accent sur la fluidité des genres et la « performativité » de l'identité (Judith Butler). Pour Butler, le genre s'affirme dans chacun des actes successifs par lesquels on se conforme à l'idéal normé du modèle masculin ou féminin. Nul ne saurait donc être pleinement homme ou femme, mais seulement tendre à le devenir. C'est pourquoi, pour Butler, le Drag Queen incarne cette dissonance entre identité intime et genre social; et c'est pourquoi, encore, le personnage d'Orlando en constitue l'une des versions. Orlando fait en effet l'expérience d'une dissociation entre identité et identification à un genre socialement déterminé par des normes. L'importance accordée au vêtement et au déguisement, tout au long du roman, traduit cette non-adhésion de l'être biologique à son genre, et l'espace de la scène permet au collectif GWEN de jouer des changements de costume pour nourrir visuellement ce propos. 
La portée philosophique de la pièce est renforcée par l'effet d'universalité produit par la jeunesse d'Orlando incarnée par celle des comédiens. Le jeu avec les anachronismes disséminés contribue à construire cette convergence des temps, par laquelle celui de Virginia Woolf rejoint le nôtre. Ainsi, entre autres exemples, le personnage de la princesse Leia de Star Wars figure parmi les tableaux des ancêtres d'Orlando projetés sur l'écran en fond de scène. Si l'adaptation rend le texte de Woolf à son épaisseur mémorielle et historique, elle transcende les époques pour produire une méditation en action sur le genre et l'identité.

Figure 2

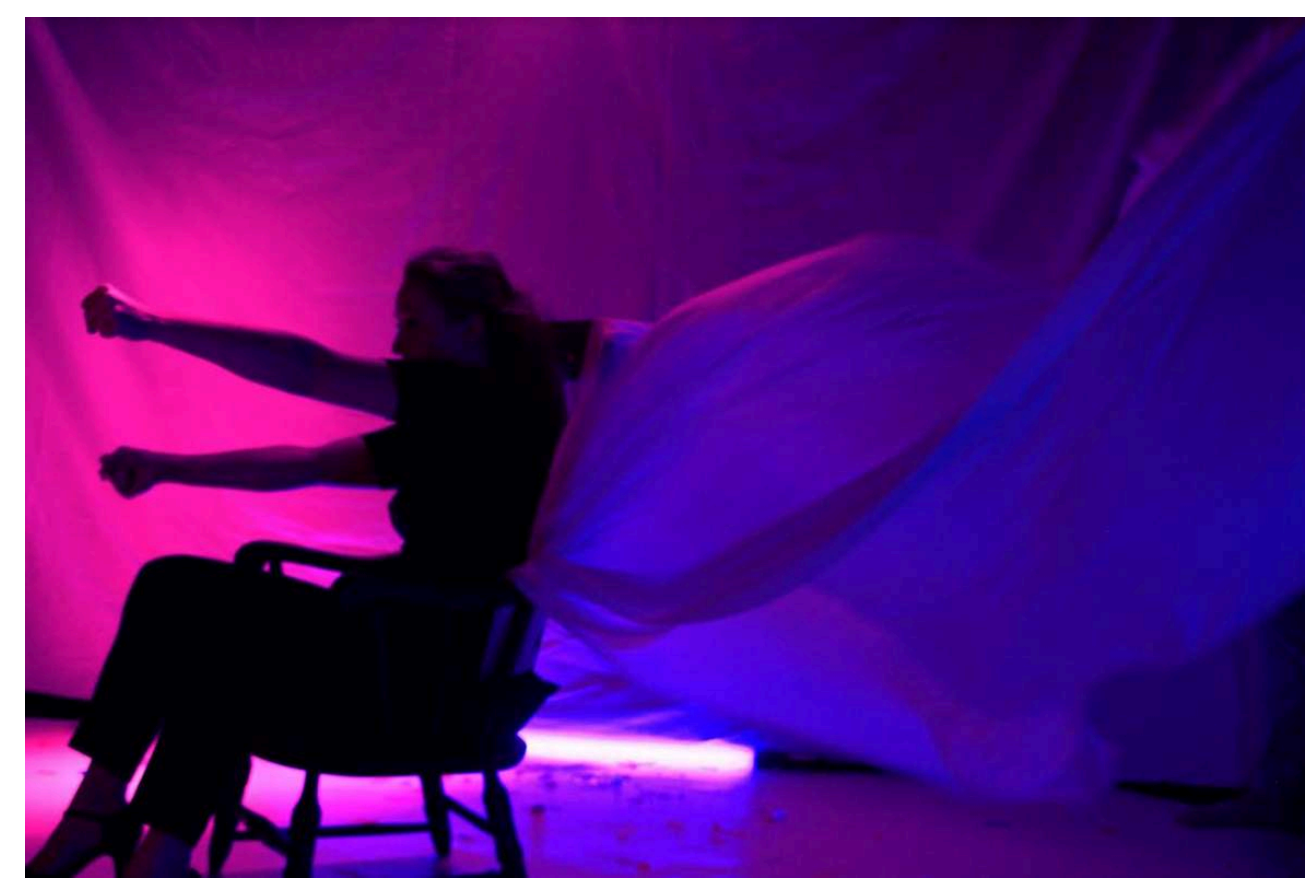

Orlando au volant de sa voiture au début du XXe siècle.

Crédits : Collectif GWEN

\section{Humour : jeux et variations sur le ton}

Cette adaptation, néanmoins, ne sombre pas dans la pesanteur métaphysique, et renoue au contraire avec la dimension fantaisiste et humoristique du roman de Woolf : «j'écris Orlando un peu comme un pastiche ", écrit Virginia Woolf dans son journal Journal (22 octobre 1928). Elle qualifie d'ailleurs son livre de "satirique dans son essence; fantastique dans sa structure " (Journal, 20 décembre 1928). Cette tonalité, qui n'est pas sans rappeler le XVIII siècle anglais et Lawrence Sterne en particulier, n'échappe pas à la critique qui parle à l'époque de «ton de moquerie» ou de "parodie». Le jeu des comédiens du collectif GWEN, autant que les nombreuses trouvailles scéniques, rendent Orlando à cet humour, qui se décline de quatre façons.

C'est d'abord la liberté du ton, qui frappe, frisant parfois l'humour potache : dans la scène 1 par exemple, la reine (jouée par un acteur) traite ouvertement Orlando comme un objet de désir dans ses gestes comme dans ses paroles; le comique, dans cette scène, est notamment produit par le jeu volontairement outrancier des acteurs, jouant avec les codes du théâtre de boulevard. 
19 Ensuite, lorsque le narrateur-biographe commente l'action, il emprunte très souvent un ton léger, comme multipliant les clins d'œil au spectateur qu'il prend à partie. Ainsi, quand il commente la disgrâce d'Orlando, suite au départ de Sasha, l'acteurcommentateur homme plaint Orlando mis à l'épreuve dans sa vie amoureuse et sociale ; au contraire, le deuxième acteur-commentateur, qui, lui, est une femme, insiste sur le fait que cette punition était bien méritée, Orlando ayant abandonné sa noble fiancée pour suivre Sasha!

20 La troisième forme de cette fantaisie déployée sur scène tient à la dérision sous laquelle tombent les codes de la masculinité dominante. En effet, dès lors qu'Orlando devient femme, chacun de ces codes est dénoncé comme relevant de l'arbitraire ou de l'idéologie, de par leur étrangeté et leur absurdité.

21 Enfin, de même que Woolf traverse quatre siècles de littérature britannique, l'adaptation multiplie les effets de pastiches théâtraux, portant l'humour dans l'imitation décalée des styles mobilisés.

\section{Traditions : jeux avec les médiums visuels}

Orlando, le roman, donne toute sa place à l'imagination et à la fantaisie grâce aux mots, ce qui permet à Woolf non seulement de s'affranchir des contraintes du récit biographique mais aussi de tourner en dérision les codes et conventions d'un tel récit. Le spectacle en fait autant et réussit à créer un univers imaginaire à partir d'une petite scène avec trois comédiens. Le spectateur n'oublie jamais qu'il est au théâtre car il n'y a aucune recherche d'illusionnisme. Au contraire, la mise en scène lui rappelle sans cesse que tout est artifice, grâce à un ensemble de dispositifs théâtraux simples mais efficaces. Au lieu de gommer les éléments les plus invraisemblables du récit, la mise en scène les accentue. Ainsi la figure mystérieuse de Sasha est-elle représentée par un drap blanc, forme ouverte à toute interprétation. La séquence où Orlando entame sa danse avec «Sasha » illustre bien comment la scène peut libérer les imaginations avec un objet banal et quelques gestes. 
Figure 3

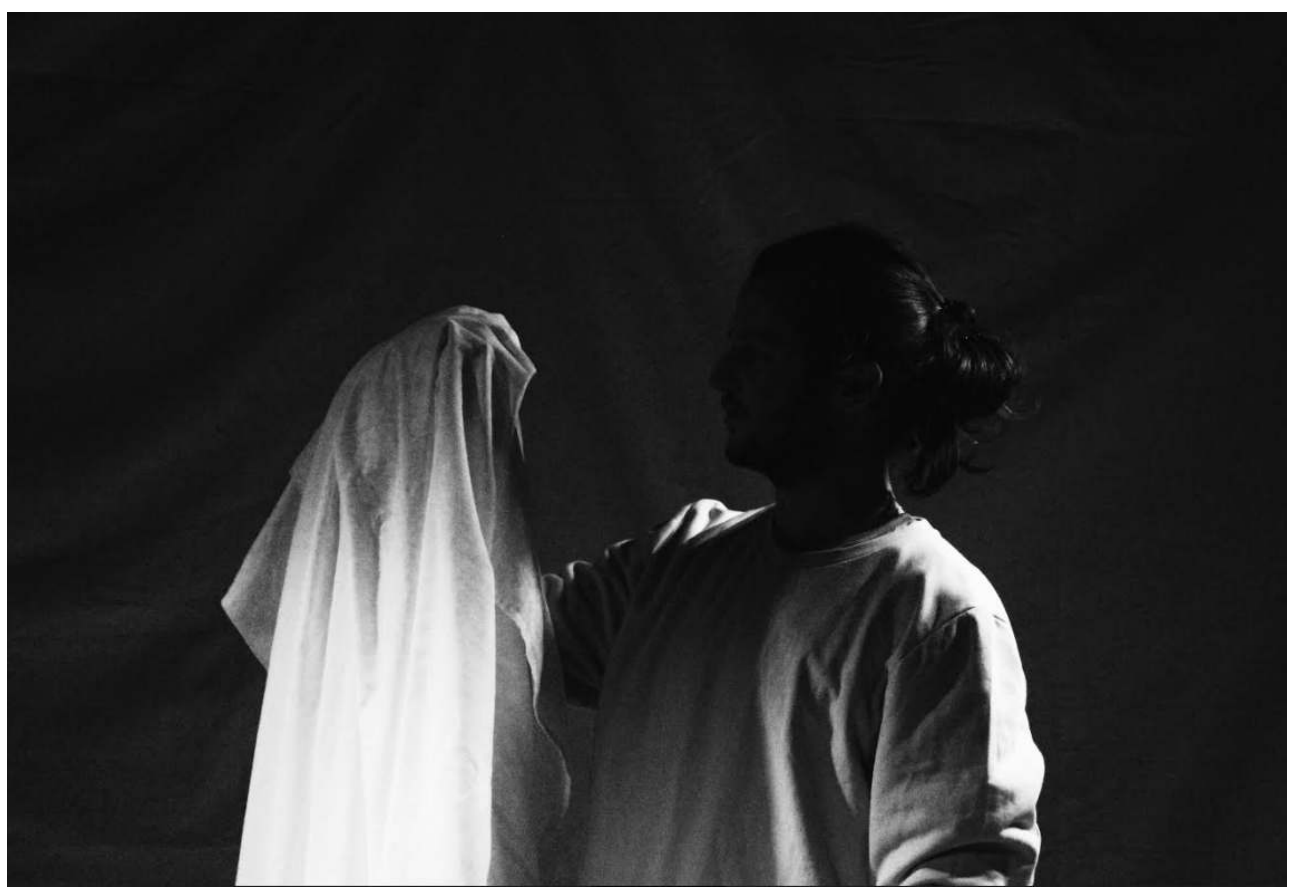

Orlando face à une Sasha aussi évanescente que le drap tenu par l'acteur.

Crédits : Collectif GWEN

Il n'y a aucune tentative non plus de rendre les transitions spatiales et temporelles moins brutales, par exemple à travers un entracte. Le roman est divisé en chapitres, mais le spectacle joue plutôt la continuité. Traditionnellement, les changements de costume se font hors scène pour que le spectateur fasse plus d'attention aux personnages joués qu'aux comédiens qui jouent. Ici les changements de costume se font sur scène et la présence des comédiens est ainsi soulignée. De cette manière, les spectateurs sont invités à s'interroger sur le lien entre costumes et rôles. La question des vêtements (masculins et féminins) revient souvent dans le roman, mais sur scène l'artifice du costume théatral permet de rendre visibles les artifices sociaux aussi. Comme à l'époque de Shakespeare, sur scène il suffit d'un vêtement et d'un objet pour que le comédien homme devienne personnage femme.

24 Ce n'est pas seulement le théâtre élisabéthain qui est évoqué ici (particulièrement approprié car on voit la Reine Elizabeth elle-même sur scène). La présence d'un narrateur qui s'adresse aux spectateurs tout au long du spectacle rappelle le théâtre de Brecht et son refus de l'illusion. Les commentaires (ironiques) du narrateur donnent aux spectateurs d'autres points de vue (parfois en contradiction avec ce que nous voyons sur scène).

Le spectacle ne se contente pas de mélanger et de parodier les différentes conventions théâtrales au fil des siècles. Certaines séquences font appel à d'autres médias. 
Figure 4



Fureur d'Orlando après le départ de Sasha. La scénographie inventive s'enrichit de la projection de textes et d'images.

Crédits : Collectif GWEN

Là où Virginia Woolf semble évoquer l'histoire de la littérature dans son roman, ce spectacle semble évoquer l'évolution d'autres façons de raconter des histoires: du théâtre au cinéma en passant par la télévision et les jeux vidéo. La partie du roman qui montre Orlando ambassadeur à Constantinople est évoquée sous la forme d'un talkshow télévisé avec une journaliste plus intéressée par la vie privée de l'ambassadeur que par sa vie publique. Quand Orlando devient femme dans la troisième partie, elle regarde (et les spectateurs aussi) un tutoriel de maquillage sur YouTube. Étant donné que le maquillage au théatre est presque aussi important que le costume (et ce depuis fort longtemps) cette séquence, au-delà de son côté comique, suggère le lien entre les rôles joués au théâtre et ceux joués dans la société.

Enfin, une scène du roman qui semble difficilement transposable sur la scène est celle à la fin du troisième chapitre où Orlando devient femme après un très long sommeil. Trois figures allégoriques, représentant la Pureté, la Chasteté et la Modestie entrent en combat contre la Vérité. Sur scène cet épisode donne lieu à une séquence qui évoque un jeu vidéo avec trois lumières. Les spectateurs les plus jeunes ont pris plaisir à reconnaître une allusion aux Pokémon, tandis que les moins jeunes ont été sensibles à la beauté féérique des jeux de lumière dans l'obscurité. Toujours est-il que le moment le plus dramatique du récit, quand Orlando devient femme, est peut-être le moment le plus magique sur le plan théâtral, comme dans un conte de fées.

\section{Conclusion}

Adapter un roman de plus de 300 pages à la scène dans un spectacle qui dure à peine une heure et quart est une gageure, comme était celle de Woolf quand elle avait choisi la forme la plus contraignante (le récit biographique) pour raconter une histoire 
insensée. La question de l'écriture et des différents styles d'écriture au long des trois siècles, n'est pas au centre de cette adaptation. En revanche, les spectateurs ont pu apprécier l'esprit espiègle de Woolf, son imagination débordante et son ton irrévérencieux qui sont tous rendus dans cette mise en scène. Les questions sérieuses posées par Woolf en 1928 de manière divertissante sont rendues tout aussi pertinentes aux spectateurs en 2020. Ce mélange du ludique et du sérieux n'est pas sans rappeler le sous-titre donné par Oscar Wilde à The Importance of Being Earnest : «A Trivial Comedy for Serious People ».

\section{RÉSUMÉS}

Critique de Orlando, pièce adaptée du roman de Virginia Woolf et mise en scène par le collectif GWEN au théâtre des Déchargeurs en janvier 2020.

Review of Orlando, a play based on Woolf's novel, adapted and directed by the collectif GWEN at Les Déchargeurs (Paris) in January 2020.

\section{INDEX}

Thèmes : Theater

Mots-clés : Orlando, Woolf, collectif GWEN, Bob Wilson, Sally Poter

Keywords : Orlando, Woolf, collectif GWEN, Bob Wilson, Sally Poter

\section{AUTEURS}

\section{SUSAN BLATTÈS}

Professeur émérite Études anglophones

Université Grenoble Alpes

Présidente RADAC (Recherches sur les Arts Dramatiques Anglophones Contemporains)

susan.blattes@univ-grenoble-alpes.fr

\section{CLAIRE HÉLIE}

Maître de Conférences

Université de Lille

Claire.helie@univ-lille.fr

\section{ANNE-LAURE RIGEADE}

PRAG Lettres modernes

Sciences Po

annelaure.rigeade@sciencespo.fr 